\title{
Biomechanical Changes at the Hip, Knee, and Ankle Joints during Gait Are Associated with Knee Osteoarthritis Severity
}

\author{
Janie L. Astephen, ${ }^{1}$ Kevin J. Deluzio, ${ }^{2}$ Graham E. Caldwell, ${ }^{3}$ Michael J. Dunbar ${ }^{4}$ \\ ${ }^{1}$ Dalhousie University School of Biomedical Engineering, 5981 University Avenue, Halifax, Nova Scotia, Canada B3H 4J5 \\ ${ }^{2}$ Queen's University Department of Mechanical and Materials Engineering, Kingston, Ontarion, Canada \\ ${ }^{3}$ University of Massachusetts Amherst Department of Kinesiology, Amherst, Massachusetts \\ ${ }^{4}$ Dalhousie University Department of Orthopedic Surgery, Halifax, Nova Scotia, Canada
}

Received 30 January 2007; accepted 5 July 2007

Published online 24 October 2007 in Wiley InterScience (www.interscience.wiley.com). DOI 10.1002/jor.20496

\begin{abstract}
Mechanical factors have been implicated in the progression of knee osteoarthritis (OA). Understanding how these factors change as the condition progresses would elucidate their role and help in developing interventions that could delay the progress of knee OA. In this cross-sectional study, we identified kinematic and kinetic variables at the hip, knee, and ankle joints that change between three clinically distinct levels of knee OA disease severity: asymptomatic, moderate OA, and severe OA. The severity level was based on a combined radiographic/symptomatic clinical decision for treatment with (severe) or without (moderate) total knee replacement surgery. Gait variables that changed between groups were categorized as: those that differed between the asymptomatic group and both OA groups, those that differed between the asymptomatic group and the severe OA group only, or those that changed progressively, that is, the asymptomatic differed from the moderate OA, and the moderate OA differed from the severe OA group. Changes seen in both OA subject groups compared to asymptomatic included increased mid-stance knee adduction moments, decreased peak knee flexion moments, decreased peak hip adduction moments, and decreased peak hip extension moments. Changes found only in the severe knee OA group included multiple kinematic and kinetic differences at the hip, knee, and ankle joints. Gait differences that progressed with OA severity included decreased stance phase knee flexion angles, decreased early stance knee extension moments, decreased peak stance phase hip internal rotation moments, and decreased peak ankle dorsiflexion moments. ( 2007 Orthopaedic Research Society. Published by Wiley Periodicals, Inc. J Orthop Res 26:332-341, 2008
\end{abstract}

Keywords: knee osteoarthritis; disease severity; gait; biomechanics; lower extremity

\section{INTRODUCTION}

Knee osteoarthritis (OA) is a metabolically active, dynamic disease that includes both destruction and repair mechanisms that may be triggered by biochemical and mechanical insults. ${ }^{1}$ No definitive cure exists, and the pathomechanics are still not well understood, ${ }^{2}$ but in the past decade gait analysis has uncovered biomechanical characteristics of knee OA. Mechanical factors such as dynamic joint loading ${ }^{3-5}$ have been implicated in knee OA pathomechanics, but their role in disease progression is equivocal. Most gait studies have characterized differences between subjects classified as either knee OA or asymptomatic. ${ }^{6-10}$ It is difficult to determine if the identified biomechanical changes were involved in the development of

Correspondence to: Janie L. Astephen (Telephone: 902-8805264; Fax: 902-494-6621; E-mail: jlasteph@dal.ca)

๑ 2007 Orthopaedic Research Society. Published by Wiley Periodicals, Inc. the disease, a response to degenerative changes in the joint and soft tissue, or a compensatory mechanism to the disease process. It is important to investigate how mechanical factors change as the condition progresses to understand the role of mechanical factors. While longitudinal studies could identify predictive factors, cross-sectional data from different levels of disease severity can provide information on which factors characterize each stage in the disease process.

Gait patterns of individuals with mild to moderate levels of knee OA have been investigated in an attempt to identify mechanical factors that are likely to be symptoms of pain and disability. ${ }^{11-14} \mathrm{In}$ particular, the magnitude of the frontal plane knee adduction moment is higher than normal in those with moderate knee $\mathrm{OA}^{11,14,15}$ and relates to radiographic severity and short-term disease progression. ${ }^{5,16,17}$ However, a comprehensive description of the biomechanical changes associated with increasing levels of knee OA severity is lacking. 
Knee OA presents a complex and multifaceted array of symptoms and risk factors, resulting in a very heterogeneous clinical condition that is difficult to classify. Most knee OA gait studies have classified subjects based on radiographic findings alone. ${ }^{5,12}$ However, there is little relationship between structural disease severity and symptoms. ${ }^{18,19}$ Clinically, a combination of radiographic and symptomatic factors is used to indicate disease severity and the need for total knee replacement, the common treatment for end-stage OA. In the present study, we used this clinical criterion to categorize disease severity, with subjects scheduled for knee replacement placed in a severe knee OA group. This assumes that candidates for knee replacement represent a distinctly different severity level than moderate knee OA patients, who were not identified as surgical candidates.

Our purpose was to identify kinematic and kinetic gait variables at the knee, hip, and ankle joints that distinguish between asymptomatic subjects and all subjects with knee OA (either moderate or severe), those that distinguish between asymptomatic and severe knee OA only, and those that change progressively from asymptomatic to moderate OA to severe OA. We hypothesized that many gait variable differences exist between the asymptomatic and severe OA groups, fewer between the asymptomatic and both OA groups, and fewer still that represent progressive changes between the three groups. From the results of previous gait studies, differences in the flexion angles at all joints, the flexion moment, the knee adduction moment, and hip adduction moment were expected. ${ }^{13,16,17}$

\section{METHODS}

\section{Subjects}

The study included 60 asymptomatic subjects, 60 with moderate knee $\mathrm{OA}$, and 61 with severe knee OA. Informed consent was obtained in accordance with the Dalhousie University Ethics Review Board. Asymptomatic subjects had no history of knee pain or surgical interventions to either lower limb and were recruited through a university news posting. They were screened for neuromuscular disorders, history of stroke and cardiovascular disease, and any other gait abnormalities.

Categorization into moderate and severe OA groups assumed that individuals indicated for total knee replacement represented a distinctly different clinical OA severity level than those who were not. Subjects indicated for joint replacement by a high-volume orthopedic surgeon within 6 months of the time of gait testing were categorized as severe; those who were not were categorized as moderate. The moderate subjects were recruited from the Orthopedic and Sports Medicine Clinic of Nova Scotia and selected from a waiting list for exploratory knee arthroscopy and from a list of individuals who had received exploratory arthroscopy surgery at least a year prior to the time of gait testing. These subjects were not candidates for knee replacement surgery. Subjects were excluded from this group if they had any major surgery or trauma to the lower limb, neuromuscular disorders, other forms of arthritis, gout, or history of stroke, or cardiovascular disease.

WOMAC self-reported pain, function, stiffness, and total scores ${ }^{20}$ were significantly greater in the severe group than the moderate and greater in the moderate group than the asymptomatic group $(p<0.0001)$. Kellgren-Lawrence (KL) radiographic scores, which assess joint severity by measurements of osteophyte presence, joint space narrowing, sclerosis, and joint deformity, ${ }^{21}$ were significantly different between the moderate and severe groups $(p<0.0001)$. The median KL score for the moderate group was 2 , and scores ranged from 1 to 4 . The median KL for the severe group was 3 , and ranged from 3 to 4 . All of the moderate subjects were either medial side dominant (medial side joint space narrowing less than lateral side joint space narrowing) or neutral (equal narrowing in both compartments). The majority (76\%) of the severe subjects had predominantly medial side disease, but most showed degenerative changes in all compartments of the knee.

\section{Gait Analysis}

Three-dimensional (3D) motion of the lower limb and external ground reaction forces of all subjects were recorded with an Optotrak 3020 optoelectronic motion capture system (Northern Digital, Inc., Waterloo, ON, Canada), and a synchronized AMTI force platform (Advanced Mechanical Technology, Inc., Watertown, MA). Three-marker triads of infrared light-emitting diodes were placed on the pelvis, thigh, shank, and foot body segments, and individual markers on the greater trochanter, the lateral epicondyle, the lateral malleolus, and the shoulder. Eight virtual markers were identified during quiet standing and used to define anatomical coordinate systems in each lower limb segment. ${ }^{22}$ Subjects were instructed to walk at a self-selected speed.

Intersegmental kinematics and kinetics were calculated by modeling the pelvis, thigh, shank, and foot as rigid bodies. The position and orientation of each segment at each time point in the gait cycle were computed using a least squares optimization routine. ${ }^{23} \mathrm{~A}$ complete gait cycle represents the time from first to second ground contact with the same foot. The 3D sign convention for the angles and moments at each joint followed a previously defined anatomically based coordinate system, ${ }^{24}$ where flexion/extension occurred about the medial/lateral axis of the proximal segment, internal/ external rotation occurred about the distal/proximal or long axis of the distal segment, and adduction/abduction occurred about the floating axis (perpendicular to the flexion/extension and internal/external rotation axes). 
Adduction/abduction and internal/external rotation angles were not examined because their relative values during gait are of a similar magnitude to that of the measurement error associated with kinematic cross-talk and skin motion. ${ }^{25-27}$ Net external intersegmental joint reaction moments were calculated using an inverse dynamics biomechanical model ${ }^{28}$ that combined positional data from the markers, ground reaction force and moment data, and limb inertial properties. ${ }^{29}$ All joint moments were normalized to body mass $(\mathrm{kg})$.

\section{Statistical Methods}

The flexion/extension angles, the net external flexion/ extension moments, ab/adduction moments, and internal/external rotation moments at the hip, knee, and ankle joints were analyzed (12 gait measures in total). These measures were represented as waveforms that changed continuously throughout the gait cycle and were defined with 101 data points, one for each percentage of the cycle.

A total of 34 discrete parameters were extracted from the 12 gait measure waveforms. These parameters included peak, minimum, and range values chosen to characterize the shape of each waveform measure. For example, the knee flexion angle waveform characteristic shape included a stance phase peak flexion angle, a swing phase peak flexion angle, and a minimum value, so two peak values, a minimum, and a range value were analyzed. A one-way ANOVA was used to investigate differences in these parameters among the three subject groups. A Bonferroni correction for 34 comparisons at an $\alpha$ level of 0.05 was used so that the effective $p$ value for each analysis was set at 0.002 for significance. A Tukey post-hoc analysis was used for multiple comparisons among the groups. Results of the ANOVA and multiple comparison analyses were categorized into three groups for interpretation purposes: all $O A$ gait changes, only severe $O A$ gait changes, and progressive gait changes. All $O A$ gait changes were those that differed between the asymptomatic group and both the moderate and severe groups. Gait changes that were only evident in the severe compared to the asymptomatic group or to the asymptomatic and moderate knee OA groups were considered only severe $O A$ changes. Gait changes were considered progressive if they increased or decreased significantly between the asymptomatic, moderate knee OA, and severe knee OA groups.

\section{RESULTS}

Demographic, stride characteristic, and WOMAC differences among the three subject groups are summarized in Table 1. Similar to knee OA patient groups of other gait analyses, both the moderate and severe knee OA groups had higher body mass index (BMI) values than the asymptomatic group. The OA groups were older than the asymptomatic group. Speed decreased incrementally from the asymptomatic to the moderate knee $\mathrm{OA}$ to the severe knee OA group; stance percentage, stride time, and stance time all increased. Stride length was smaller in the severe OA group than the asymptomatic group. All WOMAC score subscales (pain, function, stiffness, and total) were higher in the moderate group than the asymptomatic group and higher in the severe group than the moderate group, indicating more severe self-reported pain, function, and stiffness.

Significant knee, hip, and ankle differences are summarized in Tables 2, 3, and 4, respectively. All $O A$ differences at the knee joint included reduced early stance flexion moments (Fig. 1) and higher mid-stance adduction moments (Fig. 2). Both OA groups also had reduced peak and first peak hip adduction moments (Fig. 3), and reduced late stance hip extension moments. Only severe changes included reduced late stance knee extension moments (Fig. 1), reduced late stance knee internal rotation moments, and less range of sagittal plane joint motion at the knee, hip, and ankle joints. Progressive gait changes included two sagittal plane changes at the knee: successively smaller knee flexion angles during the stance phase (Fig. 4) and successively smaller knee extension moments in early stance phase (Fig. 1) from the asymptomatic group to the moderate OA group and from the moderate OA group to the severe OA group. Successively reduced late stance hip internal rotation moments and late stance ankle dorsiflexion moments were also progressive.

\section{DISCUSSION}

Several gait changes with knee OA have been reported. ${ }^{5,6,9-11}$ However, only a few investigators have attempted to capture how gait changes relate to disease progression, focusing primarily on knee adduction moment $t^{5,10,16,17}$ and, in a few cases, changes at the hip joint. ${ }^{12,13}$ In this study, we attempted to capture a more complete biomechanical description of lower extremity changes during gait that characterize different levels of disease severity. We hypothesized that the majority of differences would describe changes evident only in the severe knee OA group (only severe), but that some would characterize changes consistent with any level of severity (all $O A$ ), and some would display a trend that changes successively with severity level, suggesting the possibility of a link to a mechanism of disease progression (progressive).

As hypothesized, several only severe changes in gait variables were found, including reduced knee 
Table 1. Subject Demographic, Stride Characteristic, and WOMAC Score Comparisons between the Three Subject Groups (A=Asymptomatic, $\mathrm{M}=$ Moderate OA, $\mathrm{S}=$ Severe OA)

\begin{tabular}{|c|c|c|c|c|c|c|}
\hline \multirow[b]{2}{*}{ Parameter } & \multirow{2}{*}{$\begin{array}{c}\text { Group A } \\
\text { Mean }( \pm \mathrm{SD})\end{array}$} & \multirow{2}{*}{$\begin{array}{c}\text { Group M } \\
\text { Mean (SD) }\end{array}$} & \multirow{2}{*}{$\begin{array}{c}\text { Group S } \\
\text { Mean (SD) }\end{array}$} & \multicolumn{3}{|c|}{ Multiple Comparisons } \\
\hline & & & & M vs. A & $\mathrm{S}$ vs. $\mathrm{A}$ & $\mathrm{S}$ vs. $\mathrm{M}$ \\
\hline BMI $\left(\mathrm{kg} / \mathrm{m}^{2}\right)$ & $25.45( \pm 4.04)$ & $30.98( \pm 5.17)$ & $32.05( \pm 5.48)$ & $<0.0001$ & $<0.0001$ & 0.270 \\
\hline Weight (kg) & $73.5( \pm 14.19)$ & $93.61( \pm 17.81)$ & $91.08( \pm 15.92)$ & $<0.001$ & $<0.0001$ & 0.410 \\
\hline Age & $50.27( \pm 10.09)$ & $58.32( \pm 9.31)$ & $64.49( \pm 7.75)$ & $<0.0001$ & $<0.0001$ & $<0.0001$ \\
\hline Speed $\left(\mathrm{m} / \mathrm{s}^{2}\right)$ & $1.36( \pm 0.19)$ & $1.25( \pm 0.22)$ & $0.92( \pm 0.24)$ & 0.002 & $<0.0001$ & $<0.0001$ \\
\hline Stride length (m) & $1.44( \pm 0.13)$ & $1.39( \pm 0.16)$ & $1.16( \pm 0.19)$ & 0.073 & $<0.0001$ & $<0.0001$ \\
\hline Stride time (s) & $1.06( \pm 0.09)$ & $1.13( \pm 0.12)$ & $1.29( \pm 0.19)$ & $<0.0001$ & $<0.0001$ & $<0.0001$ \\
\hline Stance time (s) & $0.67( \pm .07)$ & $0.73( \pm 0.09)$ & $0.85( \pm 0.14)$ & $<0.0001$ & $<0.0001$ & $<0.0001$ \\
\hline Stance $\%$ & $62.8( \pm 1.59)$ & $64.23( \pm 1.90)$ & $65.64( \pm 2.26)$ & $<0.0001$ & $<0.0001$ & $<0.0001$ \\
\hline WOMAC pain & $0.44( \pm 1.41)$ & $7.53(3.94)$ & $10.62( \pm 5.82)$ & $<0.0001$ & $<0.0001$ & 0.001 \\
\hline WOMAC stiffness & $0.31( \pm 1.02)$ & $3.65( \pm 1.66)$ & $4.47( \pm 1.76)$ & $<0.0001$ & $<0.0001$ & 0.017 \\
\hline WOMAC function & $1.67( \pm 5.01)$ & $23.19( \pm 13.07)$ & $34.37( \pm 17.8)$ & $<0.0001$ & $<0.0001$ & $<0.0001$ \\
\hline WOMAC total & $2.27( \pm 6.9)$ & $34.37( \pm 17.8)$ & $50.89( \pm 17.7)$ & $<0.0001$ & $<0.0001$ & $<0.0001$ \\
\hline Gender & $\begin{array}{c}\text { Group A } \\
\text { Distribution }\end{array}$ & $\begin{array}{c}\text { Group M } \\
\text { Distribution }\end{array}$ & $\begin{array}{c}\text { Group S } \\
\text { Distribution }\end{array}$ & & & \\
\hline $\begin{array}{l}\text { Female } \\
\text { Male }\end{array}$ & $\begin{array}{l}37(62 \%) \\
23(38 \%)\end{array}$ & $\begin{array}{l}20(33 \%) \\
40(67 \%)\end{array}$ & $\begin{array}{l}33(54 \%) \\
28(46 \%)\end{array}$ & & & \\
\hline
\end{tabular}

There were significant group differences in all parameters using one-way ANOVA statistical analyses $(p<0.0001)$.

Multiple comparison $p$-values between subject groups for each parameter are shown.

Gender distribution among the subject groups is included at the bottom of the table.

Table 2. Knee Parameter Differences

\begin{tabular}{|c|c|c|c|c|}
\hline Gait Measure & Category & $\begin{array}{l}\text { Group A } \\
\text { Mean (SD) }\end{array}$ & $\begin{array}{l}\text { Group M } \\
\text { Mean (SD) }\end{array}$ & $\begin{array}{c}\text { Group S } \\
\text { Mean (SD) }\end{array}$ \\
\hline $\begin{array}{l}\text { Knee adduction moment mid-stance } \\
\text { minimum }(\mathrm{nm} / \mathrm{kg})\end{array}$ & All $O A$ & $0.21( \pm 0.09)$ & $0.29( \pm 0.13)$ & $0.31( \pm 0.22)$ \\
\hline Knee flexion moment peak (nm/kg) & All $O A$ & $0.52( \pm 0.29)$ & $0.40( \pm 0.22)$ & $0.33( \pm 0.20)$ \\
\hline Knee flexion angle peak $\left(^{\circ}\right)$ & Only Severe & $64.0( \pm 6.01)$ & $61.3( \pm 7.86)$ & $45.9( \pm 15.4)$ \\
\hline Knee flexion angle range $\left(^{\circ}\right)$ & Only Severe & $68.5( \pm 6.01)$ & $66.0( \pm 7.40)$ & $49.9( \pm 16.1)$ \\
\hline $\begin{array}{l}\text { Knee flexion moment late stance } \\
\text { minimum }(\mathrm{nm} / \mathrm{kg})\end{array}$ & Only Severe & $-0.42( \pm 0.13)$ & $-0.35( \pm 0.21)$ & $-0.10( \pm 0.19)$ \\
\hline Knee internal rotation moment peak (nm/kg) & Only Severe & $0.21( \pm 0.053)$ & $0.19( \pm 0.07)$ & $0.12( \pm 0.08)$ \\
\hline Knee flexion angle stance peak $\left(^{\circ}\right)$ & Progressive & $18.72( \pm 7.28)$ & $14.02( \pm 7.12)$ & $8.04( \pm 6.22)$ \\
\hline $\begin{array}{l}\text { Knee flexion moment early stance } \\
\text { minimum }(\mathrm{nm} / \mathrm{kg})\end{array}$ & Progressive & $-0.31( \pm 0.14)$ & $-0.26( \pm 0.10)$ & $-0.14( \pm 0.08)$ \\
\hline
\end{tabular}

Significant $(p<0.002)$ differences of one-way ANOVA on knee gait parameters are summarized, with Tukey post-hoc multiple comparison analyses.

Post-hoc analyses that indicated significant differences between both the moderate and severe OA subject groups with the asymptomatic group were considered All OA changes. Differences between the severe group and asymptomatic group (or both the asymptomatic and moderate groups) were categorized as Only Severe. Changes that increased or decreased incrementally from the asymptomatic to the moderate OA group and from the moderate OA group to the severe OA group were categorized as Progressive. 
Table 3. Hip Parameter Differences

\begin{tabular}{|c|c|c|c|c|}
\hline Gait Measure & Category & $\begin{array}{c}\text { Group A } \\
\text { Mean (SD) }\end{array}$ & $\begin{array}{l}\text { Group M } \\
\text { Mean (SD) }\end{array}$ & $\begin{array}{c}\text { Group S } \\
\text { Mean (SD) }\end{array}$ \\
\hline Hip adduction moment peak (nm/kg) & All $O A$ & $1.17( \pm 0.28)$ & $0.99( \pm 0.26)$ & $0.96( \pm 0.34)$ \\
\hline $\begin{array}{l}\text { Hip adduction moment early stance } \\
\text { peak }(\mathrm{nm} / \mathrm{kg})\end{array}$ & All $O A$ & $1.15( \pm 0.28)$ & $0.98( \pm 0.26)$ & $0.95( \pm 0.34)$ \\
\hline $\begin{array}{l}\text { Hip flexion moment late stance } \\
\text { minimum }(\mathrm{nm} / \mathrm{kg})\end{array}$ & All $O A$ & $-0.19( \pm 0.24)$ & $-0.10( \pm 0.20)$ & $-0.023( \pm 0.15)$ \\
\hline Hip flexion angle range $\left(^{\circ}\right)$ & Only Severe & $39.2( \pm 4.8)$ & $39.8( \pm 5.1)$ & $34.7( \pm 6.2)$ \\
\hline $\begin{array}{l}\text { Hip internal rotation moment stance } \\
\text { peak }(\mathrm{nm} / \mathrm{kg})\end{array}$ & Progressive & $0.11( \pm 0.09)$ & $0.074( \pm 0.09)$ & $0.031( \pm 0.04)$ \\
\hline
\end{tabular}

Significant $(p<0.002)$ differences of one-way ANOVA on hip gait parameters are summarized, with Tukey post-hoc multiple comparison analyses.

Post-hoc analyses that indicated significant differences between both the moderate and severe OA subject groups with the asymptomatic group were considered All OA changes. Differences between the severe group and asymptomatic group (or both the asymptomatic and moderate groups) were categorized as Only Severe. Changes that increased or decreased incrementally from the asymptomatic to the moderate OA group and from the moderate OA group to the severe OA group were categorized as Progressive.

extension moments (Fig. 1) and reduced knee internal rotation moments in late stance phase, as well as smaller ranges of joint motion at all three lower extremity joints and reduced peak knee flexion angles (Fig, 4). Reduced knee extension moments in late stance have been associated with knee OA in previous studies. ${ }^{30,31}$ Messier and colleagues $^{31}$ suggested that subjects with knee $\mathrm{OA}$ reduce the knee extension moment and therefore knee compressive forces by decreasing their walking speed in response to pain. This would substantiate finding this difference only in the severe knee OA population. Reduced range of motion is commonly reported as a response to the pain and dysfunction associated with degenerative joint disease, ${ }^{4,6,32}$ and reduced range of motion at all lower extremity joints has been reported. ${ }^{7}$ Individuals with more moderate disease generally have less pain and more mobility in their joints and therefore do not display the same deficiency in dynamic range of motion. Because the severe OA group consisted of individuals tested immedi- ately prior to total knee replacement, these changes were more likely to be compensatory gait responses to pain and disability and therefore less likely involved in the pathomechanics of the disease.

All $O A$ gait changes included reduced knee flexion moments in early stance (Fig. 1), which is consistent with previous studies, ${ }^{9,33,34}$ and higher mid-stance knee adduction moments (Fig. 2). Increased adduction moments are often associated with knee OA, but examination of peak adduction moments in previous studies has led to contradictory results. ${ }^{6,10}$ Our results support the recent suggestion that the mid-stance knee adduction moment is a more important parameter than the peak value for distinguishing between asymptomatic and OA gait patterns, ${ }^{15,22}$ and the mid-stance knee adduction moment is a speed-independent measure, unlike the peak knee adduction moment. $^{22}$

An additional all $O A$ change was reduced early stance peak hip adduction moments (Fig. 3).

Table 4. Ankle Parameter Differences

\begin{tabular}{|c|c|c|c|c|}
\hline Gait Measure & Category & $\begin{array}{c}\text { Group A } \\
\text { Mean (SD) }\end{array}$ & $\begin{array}{c}\text { Group M } \\
\text { Mean (SD) }\end{array}$ & $\begin{array}{c}\text { Group S } \\
\text { Mean (SD) }\end{array}$ \\
\hline Ankle flexion angle range $\left(^{\circ}\right)$ & Only Severe & $30.7( \pm 5.3)$ & $30.3( \pm 5.0)$ & $27.6( \pm 5.5)$ \\
\hline Ankle flexion moment peak (nm/kg) & Only Severe & $0.16( \pm 0.06)$ & $0.15( \pm 0.06)$ & $0.10( \pm 0.06)$ \\
\hline Ankle flexion moment minimum (nm/kg) & Progressive & $-1.39( \pm 0.11)$ & $-1.26( \pm 0.18)$ & $-1.1( \pm 0.18)$ \\
\hline
\end{tabular}

Significant $(p<0.002)$ differences of one-way ANOVA on ankle gait parameters are summarized, with Tukey post-hoc multiple comparison analyses.

Differences between the severe group and asymptomatic group (or both the asymptomatic and moderate groups) were categorized as Only Severe. Changes that increased or decreased incrementally from the asymptomatic to the moderate OA group and from the moderate OA group to the severe OA group were categorized as Progressive. 


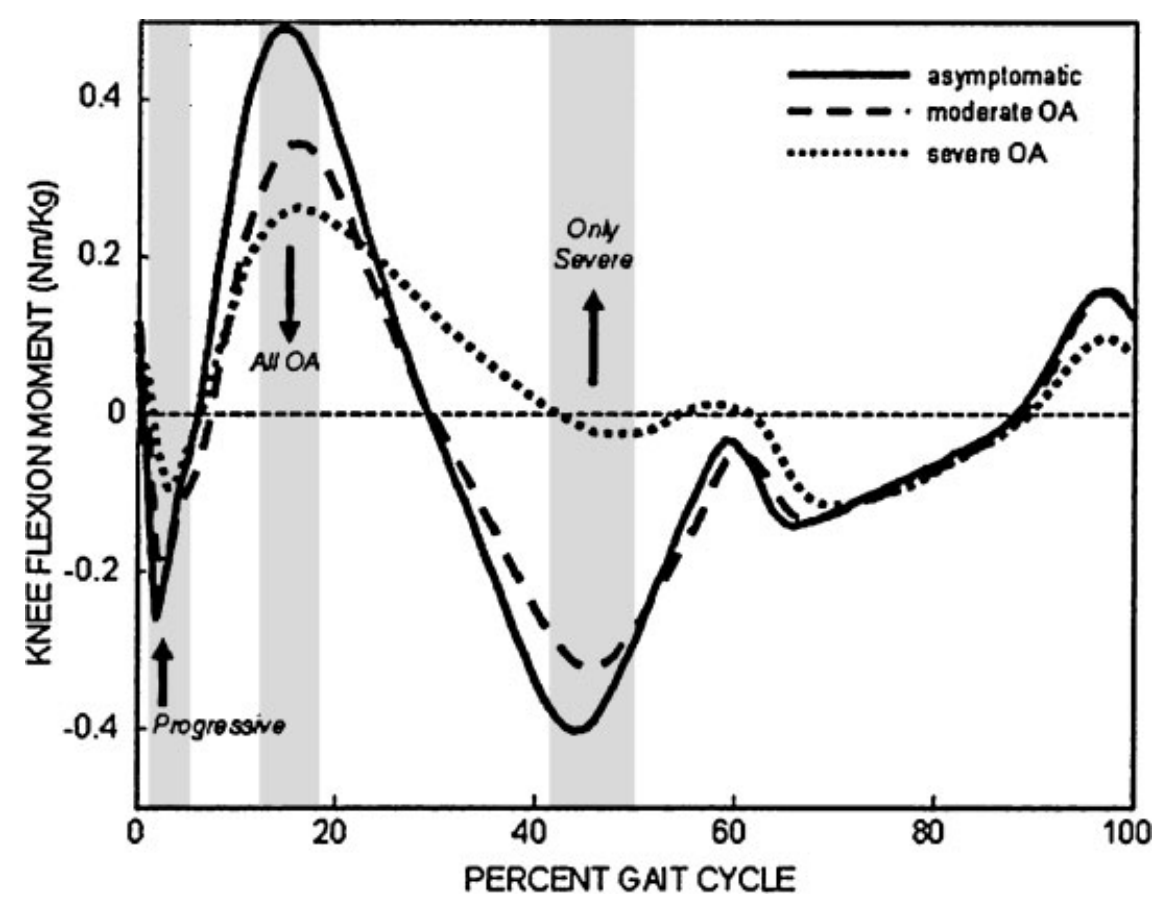

Figure 1. Knee flexion moment mean waveforms for three subject groups. Both OA groups had reduced stance phase knee flexion moments. Only the severe group had higher knee flexion moments in late stance as compared to the moderate and asymptomatic groups. Early stance phase knee extension moments were successively reduced from the asymptomatic to moderate group and from the moderate to severe group (all $p<0.002$ ).

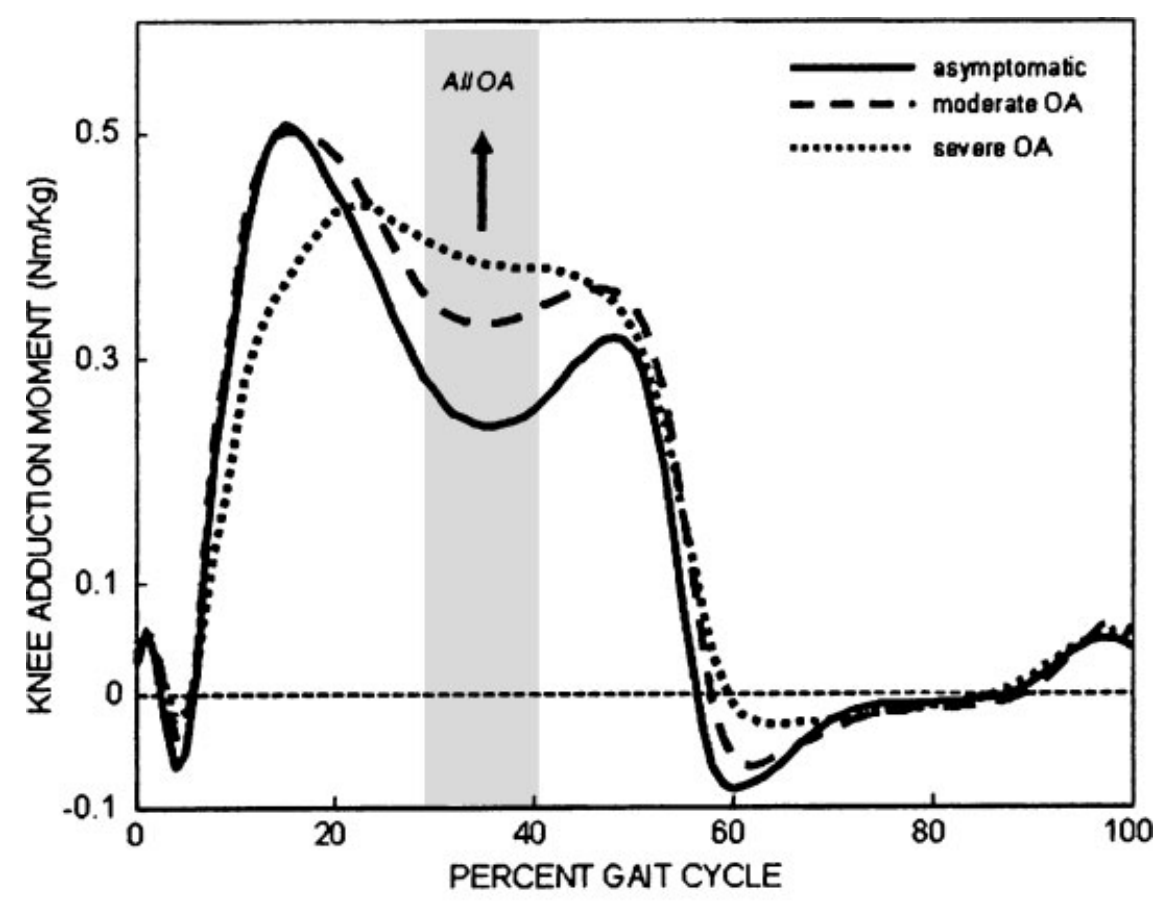

Figure 2. Knee adduction moment mean waveforms for three subject groups. Both OA groups had higher mid-stance knee adduction moments than the asymptomatic group $(p<0.002)$. 


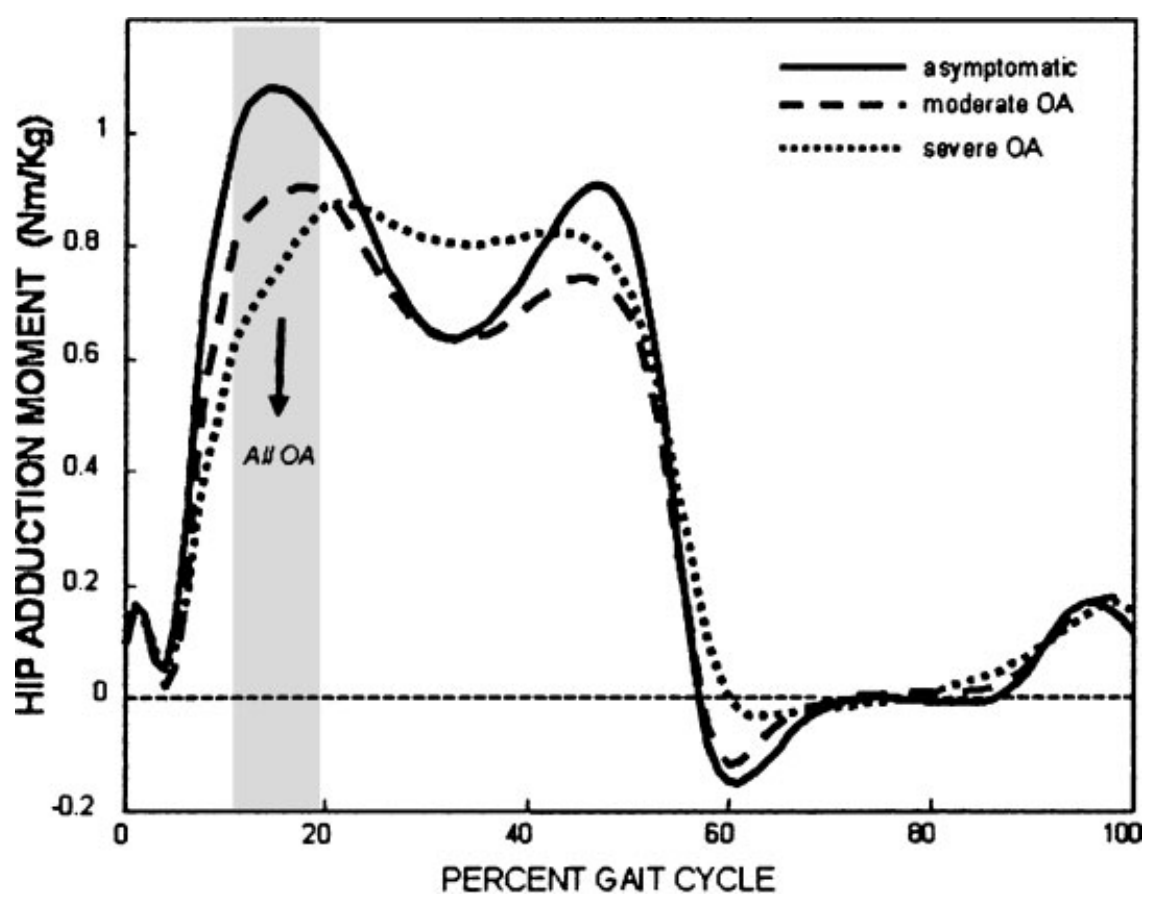

Figure 3. Hip adduction moment mean waveforms for three subject groups. Both OA groups had reduced peak hip adduction moments in early stance as compared to the asymptomatic group $(p<0.002)$.

Mundermann and colleagues ${ }^{12}$ showed that more radiographically severe OA subjects had a lower peak hip adduction moment compared to less radiographically severe OA or control subjects. Chang and colleagues ${ }^{13}$ suggested that a greater peak external hip adduction moment was associated with a decreased likelihood of knee OA progression. Direct comparison with our results is difficult, as Mundermann and colleagues categorized groups by radiographs only, and the Chang study was an 18-month follow-up study. However, both of our knee OA subject groups had reduced peak hip adduction moments, consistent with the more radiographically severe knee OA group of Mundermann and colleagues, and consistent with a more radiographically progressed disease state in Chang's study. Both OA groups also had higher late stance hip flexion moments, consistent with the results of Mundermann and colleagues. ${ }^{12}$

Reduced stance phase knee flexion angles (Fig. 4) and early stance knee extension moments (Fig. 1) were identified as progressive. While previous studies reported smaller ranges of knee flexion angle during stance with knee $\mathrm{OA},{ }^{4,6}$ our study is the first to associate changes in both peak knee flexion angle and peak extension moment just after foot contact with increasing levels of knee OA severity. Previously, we had identified a multidimensional knee OA gait factor that represented a combination of gait differences during loading response that included the knee flexion moment. ${ }^{35}$ Further, while a few knee OA gait studies have investigated changes in other lower limb joints, ${ }^{7,12,13}$ none have reported the progressive differences in hip internal rotation moment or ankle flexion moment as shown in our data. These changes occurred in late stance and may reflect a response to the altered knee mechanics detected in early stance. Gait changes categorized as progressive may be important to the pathomechanics of knee OA and warrant further investigation.

Knee OA is a multifactoral disease process that involves many interrelated factors that interact to produce biomechanical changes throughout the disease process. The present study investigated lower limb gait changes associated with knee OA, but did not establish a hierarchy or explore relationships among the identified gait factors or with other important factors such as neuromuscular control, BMI, and walking velocity. For example, obesity is one of the most common risk factors of knee OA, ${ }^{36}$ and the OA subject groups had higher BMI levels that increased with severity (Table 1). In this study and others, joint moments were normalized to body mass to account for subject differences. As the majority of Canadians with knee $\mathrm{OA}$ are overweight or obese ${ }^{37}$ it is difficult to control for BMI effects in the design of knee OA gait 


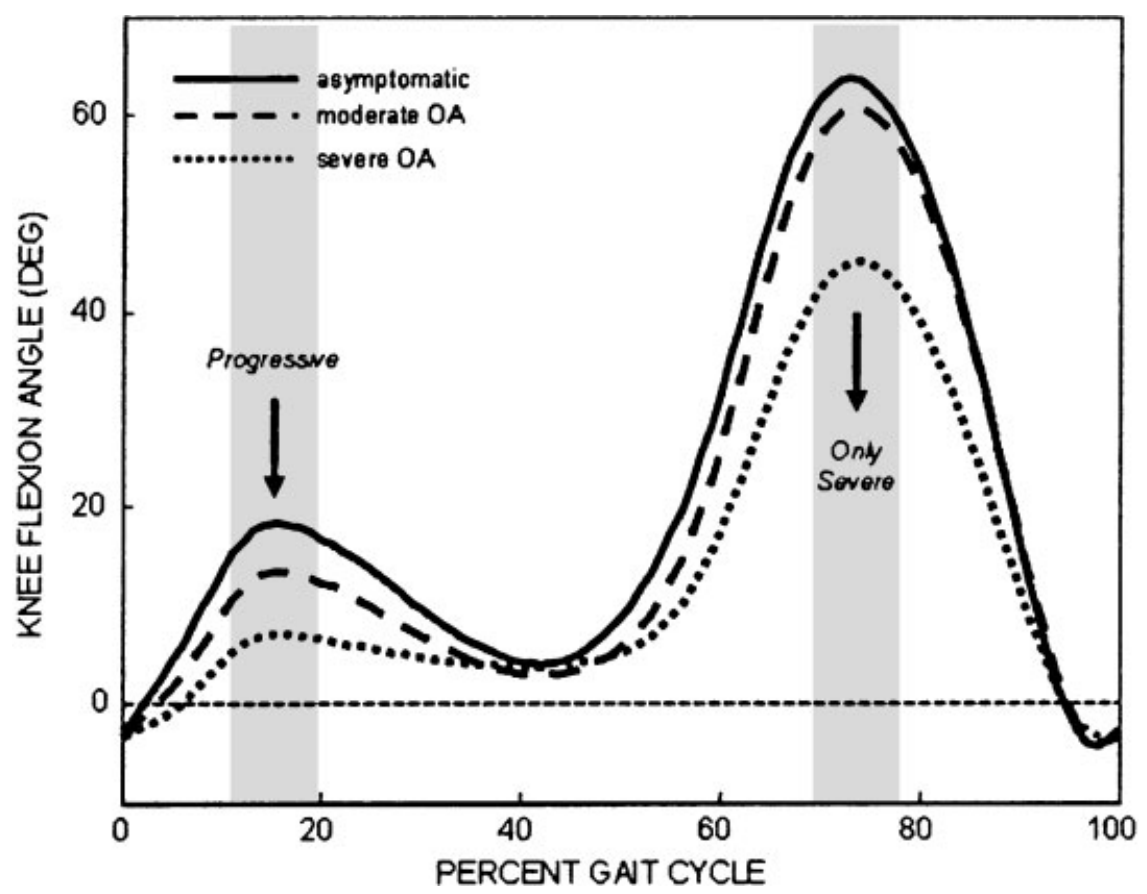

Figure 4. Knee flexion angle mean waveforms for three subject groups. Peak knee flexion angles in stance were reduced successively from the asymptomatic to the moderate group and from the moderate group to the severe group. The severe group had reduced peak knee flexion angles as compared to the asymptomatic and moderate groups (all $p<0.002$ ).

studies. Some previous studies have shown that obese individuals use altered gait mechanics ${ }^{38}$ and walk with increased total energy expenditure. ${ }^{39}$ However, some obese individuals may be able to reorganize neuromuscular function during gait, enabling them to maintain joint health, ${ }^{38}$ so the effect of obesity on joint mechanics and neuromuscular control warrants further study.

As in other knee OA studies, ${ }^{6,40,41}$ the subjects in the present study walked slower than the asymptomatics, and speed was reduced successively among the three severity groups (Table 1). Previous studies have demonstrated the effect of walking speed on gait measures, particularly in the sagittal plane. ${ }^{33,42,43}$ While gait speed causes biomechanical changes in asymptomatic individuals, no evidence exists of a clear causal path between gait speed and gait mechanics in the presence of knee OA. Gait speed is inherently linked to the disease process of knee OA, making it difficult to separate the two confounding effects. Some have attempted to account for the confounding effect of gait speed by selecting and comparing gait trials at a speed close to $1 \mathrm{~m} / \mathrm{s} \cdot{ }^{5,9,10}$ However, this approach requires OA subjects to walk faster than they normally would, or asymptomatic subjects to walk slower than they prefer, or both. We wanted subjects to walk at their self-selected speed to characterize the natural mechanical environment of the joints during a daily activity. Another approach is to remove the effect of speed statistically with an analysis of covariance (ANCOVA) model. This was inappropriate in our study, because critical ANCOVA assumptions are violated when the covariate (speed) is affected by the treatment (OA). ${ }^{44-46}$ ANCOVA is also contraindicated when large differences exist in the mean and range values of the covariate between groups, ${ }^{44,47,48}$ as shown in Table 1.

We extracted discrete parameters from the gait waveform profiles, but differences in the patterns of waveforms may provide more information on gait changes with knee OA severity. We previously developed methodology for extracting and comparing characteristic patterns of gait waveforms ${ }^{49}$ and for examining relationships between gait measures. ${ }^{50}$ Further study will use these techniques to identify multifactoral changes in gait waveforms that relate to disease severity. Finally, although we report differences among three clinically distinct subject groups, severity levels within each OA group covered a spectrum of disease both in terms of radiographs and symptoms. Investigation of how the biomechanical factors identified in this study relate to the spectrum of radiographic, pain, and functional measures should provide further insight to how these gait factors relate to disease progression. 


\section{ACKNOWLEDGMENTS}

This research was supported by the Natural Sciences and Engineering Research Council of Canada.

\section{REFERENCES}

1. Felson DT, Lawrence RC, Dieppe PA, et al. 2000. Osteoarthritis: new insights. Part 1: the disease and its risk factors. Ann Intern Med 133:635-646.

2. Brandt KD. 1998. The importance of nonpharmacologic approaches in management of osteoarthritis. Am J Med 105:39S-44S.

3. Radin EL, Yang KH, Riegger C, et al. 1991. Relationship between lower limb dynamics and knee joint pain. J Orthop Res 9:398-405.

4. Messier SP, Loeser RF, Hoover JL, et al. 1992. Osteoarthritis of the knee: effects on gait, strength, and flexibility [published erratum appears in Arch Phys Med Rehabil 1992;73:252]. Arch Phys Med Rehabil 73: 29-36.

5. Sharma L, Hurwitz DE, Thonar EJ, et al. 1998. Knee adduction moment, serum hyaluronan level, and disease severity in medial tibiofemoral osteoarthritis. Arthritis Rheum 41:1233-1240.

6. Kaufman KR, Hughes C, Morrey BF, et al. 2001. Gait characteristics of patients with knee osteoarthritis. J Biomech 34:907-915.

7. Al Zahrani KS, Bakheit AM. 2002. A study of the gait characteristics of patients with chronic osteoarthritis of the knee. Disabil Rehabil 24:275-280.

8. Hilding MB, Lanshammar H, Ryd L. 1995. A relationship between dynamic and static assessments of knee joint load. Gait analysis and radiography before and after knee replacement in 45 patients. Acta Orthop Scand 66:317320.

9. Baliunas AJ, Hurwitz DE, Ryals AB, et al. 2002. Increased knee joint loads during walking are present in subjects with knee osteoarthritis. Osteoarthritis Cartilage 10:573579 .

10. Hurwitz DE, Ryals AB, Case JP, et al. 2002. The knee adduction moment during gait in subjects with knee osteoarthritis is more closely correlated with static alignment than radiographic disease severity, toe out angle and pain. J Orthop Res 20:101-107.

11. Landry SC, Mckean KA, Hubley-Kozey CL, et al. 2007. Knee biomechanics of moderate OA patients measured during gait at a self-selected and fast walking speed. Journal of Biomechanics 40:1754-1761.

12. Mundermann A, Dyrby CO, Andriacchi TP. 2005. Secondary gait changes in patients with medial compartment knee osteoarthritis: Increased load at the ankle, knee, and hip during walking. Arthritis Rheum 52:2835-2844.

13. Chang A, Hayes K, Dunlop D, et al. 2005. Hip abduction moment and protection against medial tibiofemoral osteoarthritis progression. Arthritis Rheum 52:35153519 .

14. Lewek MD, Rudolph KS, Snyder-Mackler L. 2004. Control of frontal plane knee laxity during gait in patients with medial compartment knee osteoarthritis. Osteoarthritis Cartilage 12:745-751.

15. Weidenhielm L, Svensson OK, Brostrom LA, et al. 1994. Adduction moment of the knee compared to radiological and clinical parameters in moderate medical osteoarthrosis of the knee. Ann Chir Gynaecol 83:236-242.

16. Mundermann A, Dyrby CO, Hurwitz DE, et al. 2004. Potential strategies to reduce medial compartment loading in patients with knee osteoarthritis of varying severity: reduced walking speed. Arthritis Rheum 50:1172-1178.

17. Miyazaki T, Wada M, Kawahara H, et al. 2002. Dynamic load at baseline can predict radiographic disease progression in medial compartment knee osteoarthritis. Ann Rheum Dis 61:617-622.

18. Hannan MT, Felson DT, Pincus T. 2000. Analysis of the discordance between radiographic changes and knee pain in osteoarthritis of the knee. J Rheumatol 27:15131517.

19. Dieppe PA. 2005. Relationship between symptoms and structural change in osteoarthritis: what are the important targets for therapy? J Rheumatol 32:1147-1149.

20. Bellamy N, Buchanan WW, Goldsmith $\mathrm{CH}$, et al. 1988. Validation study of WOMAC: a HealthStatus instrument for measuring clinically important patient-relevant outcomes following total hip or knee arthroplasty in osteoarthritis. J Orthop Rheum 1:95-108.

21. Kellgren J, Lawrence J. 1957. Radiographic assessment of osteoarthritis. Ann Rheum Dis 16:494-501.

22. Landry SC, Mckean KA, Hubley-Kozey CL, et al. 2006. Knee biomechanics of moderate OA patients measured during gait at a self-selected and fast walking speed. Journal of Biomechanics 40:1754-1761.

23. Challis JH. 1995. A procedure for determining rigid-body transformation parameters. J Biomech 28:733-737.

24. Grood ES, Suntay WJ. 1983. A joint coordinate system for the clinical description of three- dimensional motions: application to the knee. J Biomech Eng 105:136-144.

25. Kadaba MP, Ramakrishnan HK, Wooten ME. 1990. Measurement of lower extremity kinematics during level walking. J Orthop Res 8:383-392.

26. Piazza SJ, Cavanagh PR. 2000. Measurement of the screwhome motion of the knee is sensitive to errors in axis alignment. J Biomech 33:1029-1034.

27. Reinschmidt C, van den Bogert AJ, Nigg BM, et al. 1997. Effect of skin movement on the analysis of skeletal knee joint motion during running. J Biomech 30:729-732.

28. Costigan PA, Wyss UP, Deluzio KJ, et al. 1992. A semiautomatic 3D knee motion assessment system. Medical and Biological Engineering and Computing 5:343-350.

29. Clauser CE, McConville JT, Young JW. 1969. Weight, volume and center of mass of segments of the human body. AMRL Tech Rep 60-70.

30. Gok H, Ergin S, Yavuzer G. 2002. Kinetic and kinematic characteristics of gait in patients with medial knee arthrosis. Acta Orthop Scand 73:647-652.

31. Messier SP, Gutekunst DJ, Davis C, et al. 2005. Weight loss reduces knee-joint loads in overweight and obese older adults with knee osteoarthritis. Arthritis Rheum 52:2026 2032.

32. Childs JD, Sparto PJ, Fitzgerald GK, et al. 2004. Alterations in lower extremity movement and muscle activation patterns in individuals with knee osteoarthritis. Clin Biomech (Bristol, Avon) 19:44-49.

33. Hurwitz DE, Ryals AR, Block JA, et al. 2000. Knee pain and joint loading in subjects with osteoarthritis of the knee. J Orthop Res 18:572-579.

34. McAlindon TE, Cooper C, Kirwan JR, et al. 1993. Determinants of disability in osteoarthritis of the knee. Ann Rheum Dis 52:258-262. 
35. Astephen JL, Deluzio KJ. 2005. Changes in frontal plane dynamics and the loading response phase of the gait cycle are characteristic of severe knee osteoarthritis application of a multidimensional analysis technique. Clin Biomech (Bristol, Avon) 20:209-217.

36. Felson DT, Zhang Y, Hannan MT, et al. 1997. Risk factors for incident radiographic knee osteoarthritis in the elderly: the Framingham Study. Arthritis Rheum 40: 728-733.

37. 2006 CJRR report: total hip and total knee replacements in Canada. Ottawa, Ontario, Canada: Canadian Institute for Health Information.

38. DeVita P, Hortobagyi T. 2003. Obesity is not associated with increased knee joint torque and power during level walking. J Biomech 36:1355-1362.

39. Browning RC, Baker EA, Herron JA, et al. 2006. Effects of obesity and sex on the energetic cost and preferred speed of walking. J Appl Physiol 100:390-398.

40. Olney SJ, Griffin MP, McBride ID. 1994. Temporal, kinematic, and kinetic variables related to gait speed in subjects with hemiplegia: a regression approach. Phys Ther 74:872-885.

41. Mattsson E, Brostrom LA, Linnarsson D. 1990. Changes in walking ability after knee replacement. Int Orthop 14: $277-280$.
42. Kirtley C, Whittle MW, Jefferson RJ. 1985. Influence of walking speed on gait parameters. J Biomed Eng 7:282-288.

43. Lelas JL, Merriman GJ, Riley PO, et al. 2003. Predicting peak kinematic and kinetic parameters from gait speed. Gait Posture 17:106-112.

44. Milliken GA, Johnson DE. 2002. Analysis of messy data volume III: analysis of covariance. New York: Chapman and Hall/CRC.

45. Cox DR, Mccullagh P. 1982. Some aspects of analysis of covariance. Biometrics 38:541-554.

46. Cochrane WG. 1957. Analysis of covariance: its nature and uses. Biometrics 13:261-281.

47. Smith HF. 1957. Interpretation of adjusted treatment means and regressions in analysis of covariance. Biometrics 13:282-308.

48. Howell DC. 1997. Statistical methods for psychology, 4th ed. Belmont, CA: Wadsworth.

49. Deluzio KJ, Astephen JL. 2007. Biomechanical features of gait waveform data associated with knee osteoarthritis: an application of principal component analysis. Gait Posture 25:86-93.

50. Astephen JL, Deluzio KJ. 2004. A multivariate gait data analysis technique: application to knee osteoarthritis. Proc Inst Mech Eng [H] 218:271-279. 